\title{
A Tourism Resources Integration Mechanism based on XML and Web Service
}

\author{
Tao $\mathrm{Hu}^{1}$, Weicai $\mathrm{Du}^{2}$, and Linfeng Dong ${ }^{3}$ \\ Information Management Department, \\ Tourism College, Hainan University, \\ 58 Renmin Ave, Haikou, Hainan, 570228, P. R. China \\ ' huxuefeng2000@yahoo.com, ${ }^{2}$ dwencai@hotmail.com, ${ }^{3}$ dlfphd@163.com
}

\begin{abstract}
To tackle problems such as isolated data islands in tourism as so much assorted data should be processed, it is constructed a platform that can share data and information of tourism resources. XML and Web Services are getting more and more attentions in many fields such as enterprise, egovernment and so on as their great expressive ability in data and services. In this paper, the integrated tourism data processing platform based on XML and Web Services is described. Compared with others, it is efficient as semistructured data description is convenient and simple for data processing, sharing and exchanging. Then, a case study is described to illustrate how the integration mechanism can be applied in practice. At last, some open issues and future work are discussed.
\end{abstract}

\section{Introduction}

Tourism is a mushroom industry today, but how to use tourism resources reasonably and efficiently to develop tourism industry in a more profitable way; and increase the ratio of tourism resources usage [1] are the key problems confront us. The degree of using information technology in many enterprises is not high, and some one are only portal web site of their company, lacking of basic business service such as transaction processing system, management information system, not to mention decision support system. One reason is that the information platform is designed on independent planning and isolated implementation mode, there existing much differences in data format, data storage and system platform between so many departments, and it may be very difficult to implement data sharing and exchanging

Please use the following format when citing this chapter:

Hu, T., Du, W., Dong, L., 2006, in International Federation for Information

Processing, Volume 205, Research and Practical Issues of Enterprise Information Systems, cds. Tjoa, A.M., Xu, L., Chaudhry, S., (Boston:Springer), pp.407-415. 
with each other for so much assorted legacy systems with people's not inclination to change them.

With this situation, it is very important to develop an open application platform for data exchanging and collaborative application across several business domains. This schema is not an application infrastructure which is only used for present data integration and sharing, and it is an integration standards and solutions for data exchanging which are compatible for all relative tourism organizations. Any application, if its application schema is based on the normalizations, which have been designed for all entities such as enterprise, government or travel agency, it may need only some degree of alternation of existing application for applying into the whole collaborative system, which could share information and exchange data resources.

Web Service that is based on SOAP (Simple Object Access Protocol) represents the future application direction of distributed computing technology. Combined with $\mathrm{XML}$, Web service consists of so many distinguishing features such as operating system independent, high efficiency and convenience in use, and the ability of communications across platform and firewall [2-4].

Under this computing environment, all resources published in standard description language are distributed, and it is a real collaborative platform with the advantages of platform independent. And this paper is under the research about collaborative environment based on XML and Web Service.

The outline of tourism resources integration could be drawn as following: the infrastructure of collaborative work based on Web Service technology is presented first; then the ontology of tourism information resources based on main techniques and standards such as SOAP, XML, UDDI, WSDL and WSLF is described [5-7]; on the basis of these, tourism information resources are integrated, and the standards of various tourism resources are given. At last, it will be realized the goal of unified standards, united construction, information complementation and data shares.

On the research of Web Service and workflow [8-10], they are integrated into applications in tourism seamlessly. As the undividable part of web service infrastructure, XML is integrated into the web service collaborative workflow and supplying information service for the whole collaborative platform. Therefore, it completes the data sharing and information exchange of tourism information resources; accelerates the development of information intelligent island, and realizes the agility, standardization and normalization of information service.

The rest of this paper is structured as follows. Section 2 will give background information about tourism information resources sharing and exchanging. In section 3 we will describe how tourism information resources can be integrated based on $\mathrm{XML}$ and Web Service. Section 4 will give a case study, which illustrates how the integration mechanism can be applied in practice. Finally, in Section 5 we will discuss some open issues and future work, and conclude the paper. 


\section{Background}

There is a need for tourism resources web services based on $\mathrm{xml}$ when applying search about a travel scenario to get the tourism information such as air, spot, hotel etc and save it to local database for private use. In fact, it is not easy to complete this task as all the information given about the research may be described in some different way, and it is not convenient to process for local use. For example, if search "hotel", "jiuzhaigou", "phone", the information found may be expressed in some different way and format. Some provides the zone code such as "0837-7734xxx", and some other may not provide it as " $7734 \mathrm{xxx".} \mathrm{On} \mathrm{the} \mathrm{other} \mathrm{way,} \mathrm{this} \mathrm{information}$ is described in web page as a text format that is not structured and not convenient for local use such as saving and processing. In addition to this, the same data may be drawn in several methods. For example, phone may be given as "phone" or "telephone" and this will bring misuse and promiscuous. If all hotels present their information in standard way, e.g. phone use telephone to present the number by a web service and all the information is shown in a structure way such as XML, it will be simple and easy for using and processing.

Lara mentions web services oriented specifications such as DAML-S, OWL-S and industry web services standards such as WSFL, BPELAWS, and BPML [11]. Singh [12] discusses the workflow composition of services as a community of services providing using shared information in a convenient way. Paper [13] gives UDDI registry that can be used in Hotel Reservation registering their offerings as web services. It is investigated that integrating web service using the basic workflow patterns as defined by van der Aalst [14]. On-line businesses are beginning to adopt a developmental paradigm where high-level component-based services and semantic web services [15] are becoming sufficiently modular and autonomous to be capable of fulfilling the requirements of other businesses. In paper [16], it is described that Input/Output Messages of multiple services should be transformed into independent ontology, and then consensus ontology is used to help integrate these messages. This consensus ontology is decomposed into the Value_Names. Thus services incorporate the integrated knowledge that may be used by other entity. Zeng [17] conducts research that uses Unified Modeling Language (UML) state charts and agentoriented methods for declarative peer-to-peer service composition that is useful for web services to be integrated into a composite one providing a unified service.

Later in this paper we will show how can integrate heterogeneous legacy system into a standard one in data format and to be easy for information communication and exchange.

\section{Tourism information resources integration based on XML and Web Service}

As the key technology for multi-point data exchange based on Internet, XML is very suitable to interchange business data across many sorts of enterprises/applications. 
Some companies such as IBM, Microsoft, and DevelopMentor etc collaboratively constituted Simple Object Access Protocol (SOAP) specifications. SOAP is based on $\mathrm{XML}$ and maintains the openness and extensibility of description. It used application protocols based TCP/IP such as HTTP, SMTP, IMAP4 and POP3 etc, and be compatible with present communication infrastructure.

As Web protocols are standard, the language to be used for program and the platform the web service is running on are not important. Using HTTP protocols to communicate applications can across firewall without affecting it in low degree. It also provides the interactive communications with other services over network.

$\mathrm{XML}$ is discovered to be excellent and much suitable for B2B applications. B2B e-commerce depends on frequent exchanging of business information between many enterprises, and it flows smoothly between various enterprise applications. And completely developed B2B e-information flow can reduce greatly the degree of human working. In addition to this, it can improve the efficiency of transactions and obtains more business opportunities for enterprises.

Data exchange center is fully based on Web Service and. SOAP as the basis of secure communication, XML as the technology of data exchange across platforms, .net as the technology of application realization across platforms, and set up the data share standards for each level of tourism applications.

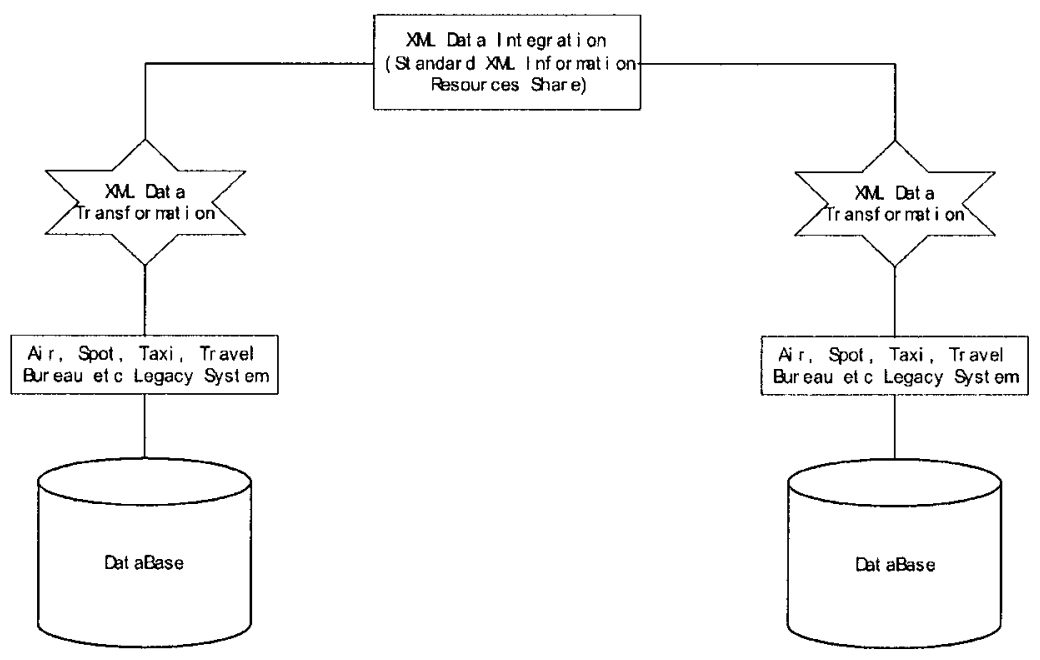

Fig. 1. Travel information resources data integration by XML

Fig 1 shows the diagram of tourism information sharing flows. The system consists of the follows. Service provider presents their services in Internet that can be searched by customers. When a user selects the service, his or her requests will be sent in XML format by SOAP to the service provider. And this service and client's request are described in standard $\mathrm{xml}$ format. As provider may use his own legacy system which service is drawn in private ontology that is not compatible with 
standard $\mathrm{xml}$ format. Therefore, the request expressed in standard $\mathrm{xml}$ format will be translated into local xml format by xml-mapping.

Then, the service provider application executes the query by customer's demands that are now given in local xml format. When this completed, the result of query is presented in local xml format. If it is prompted to customer, he or she may not recognize it cause of different $\mathrm{xml}$ format contained different ontology. Thus, the request should be translated into standard $\mathrm{xml}$ format by xml-mapping again. The result of query that meets customer's demands will be responded to customer in standard $\mathrm{xml}$ format by SOAP, and formatted in XSLT to be in standard display format. As in standard $\mathrm{xml}$ format, the user can understand the information easily and convenient for his/her use.

When the user gets the information in standard $\mathrm{xml}$ format, and he/she can store the query result in his or her private $\mathrm{xml}$ format for future use. As the result is in standard xml format, it should be translated into the customer's local xml format by xml-mapping. Thus, it is compatible with the user's legacy system.

It consists of the following parts:

- WebServices 10

Inputs: $\mathrm{xml}$ request based on standard request $\mathrm{xml}$ schema;

Outputs: xml response based on standard response xml schema;

- WebApplication1()

Inputs: user input information,

Process: invocating the corresponding web service, and get the show information to be displayed

Outputs: shows request response information.

The data flow is as follows:

Customer's request is in:

$\mathrm{XML}$ request document and schema based on Standard schema;

After strand->local XML-Mapping, it is shown in:

XML request document and schema based on Local schema

Carrying out query and finished, the result will be described in:

XML response document and schema based on Local schema

After local-standard XML-Mapping, it is shown in:

$\mathrm{XML}$ response document and schema based on standard schema

- WindowsApplication2

Inputs: XML response document and schema based on standard schema 
Process: transfer the document to be xml document based on local schema, and write to local database;

Outputs: xml document based on local schema

The information exchange and share center is based on XML and Web service as their semi-structured data expressive ability and convenient way in usage. And their ubiquitous services bring more and more organizations to join to this platform. On the basis of it, one-more, more-one and more-more sharing mode takes effect. It will be widely used in tourism for its service platform that integrates tourism data and tourism services which as shown in Fig. 2.

Fig. 2, the travel information resources share platform integrates information of hotel, airlines, travel agency, taxi, traveler, spot and travel bureau together and implements the data sharing and information exchanges. It is the tourism data sharing and exchanging center. Information provided by any entity (e.g. a Hotel) can be used in horizontal industrial networks (other hotels) or vertical industrial networks (travel bureau, airlines, travel agency, traveler etc).

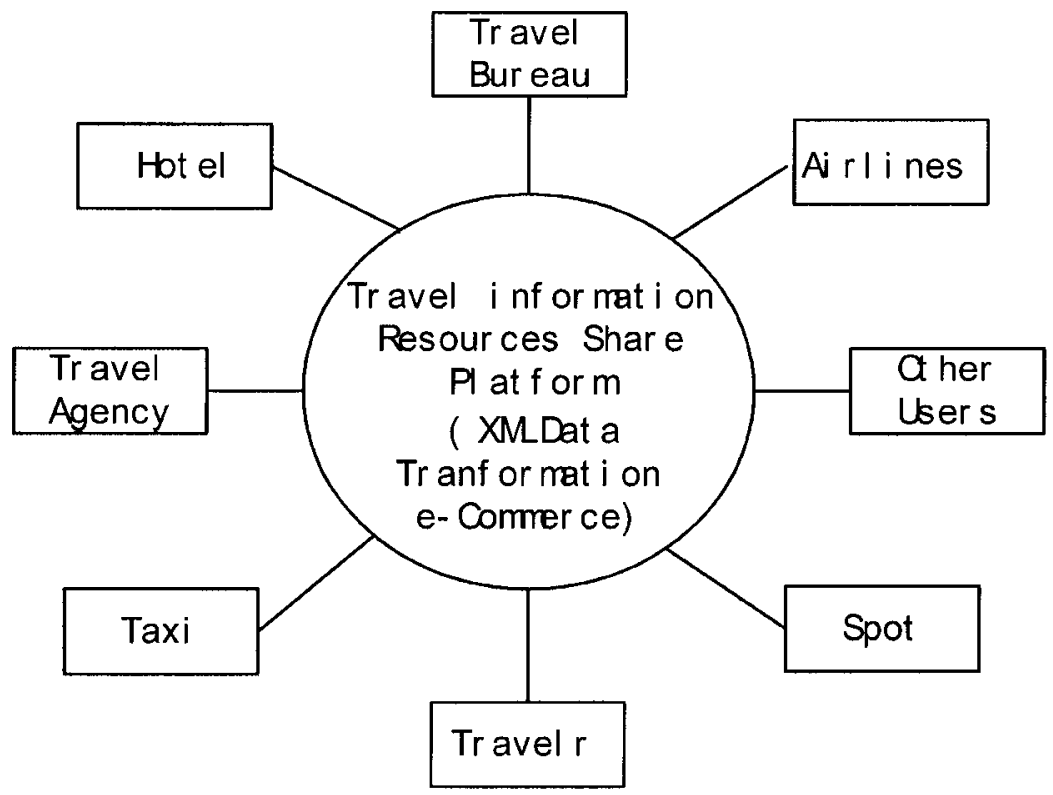

Fig. 2. Travel information resources share platform 


\section{Example scenario}

We develop an application system shown in Fig. 3. Customers visit the hotel web service page and send their request. The request is formatted in xml standard document and schema. Before sending to the background web application1 for performing the request, it can be prompted to customer in XSLT for confirming his or her request. Then the web application 1 will do xml-mapping translating request $\mathrm{xml}$ document in standard $\mathrm{xml}$ schema to request xml document in local xml schema; performing the customer's request - querying the provider's database to get the response document expressed in local response xml schema; and do second xmlmapping translating response $\mathrm{xml}$ document in local $\mathrm{xml}$ schema to response $\mathrm{xml}$ document in standard xml schema.

The web applicationl (Containing web service application) will send response $\mathrm{xml}$ document to the customer and displayed by XSLT. The customer can do xmlmapping translating response $\mathrm{xml}$ document in standard $\mathrm{xml}$ schema to response $\mathrm{xml}$ document in his/her local xml schema, and saves it to customer's database for future use.

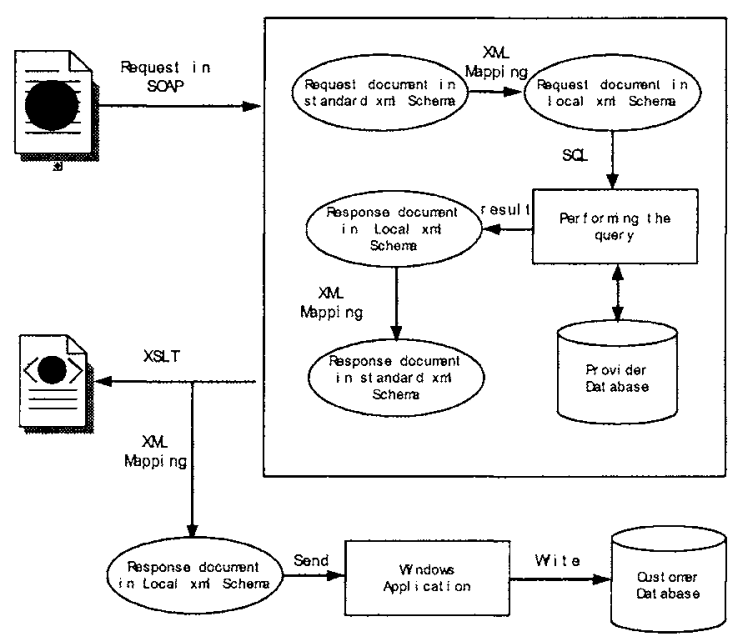

Fig. 3. Tourism resources integration based on XML and Web Service

\section{Conclusions}

As tourism is developing in mushroom way, it grows up accompanying multiassorted data such as air, hotel, taxi, and so on. In order to use these information resources, an integration platform based on $\mathrm{xml}$ and web service is constructed with 
the aid of MS Visual Studio net. Compared with others, it realizes information sharing and exchanging, and be convenient to be used. At the future research, we focus on the integration mode with the adaptive workflow to cooperate with all relative tourism organizations such as hotel, air, and scene spot for data sharing and information exchanging. In addition to these, it may be another valuable research direction tourism information change management.

\section{Acknowledgments}

This research is supported by the National Science Fund No. 70561001/G0110 and the Province Science Fund Project "the Research on the collaboration security pattern in e-Government".

\section{References}

1. L.C. Wei, Struggling for setting up a prosperous travel province of Hainan -a speech in the whole travel industry development conference (May 28, 2004); http://www.wstt.com/Information/colligate/20040605/101154.html.

2. R.J. Glushko, J.M. Tenenbaum, and B. Meltzer, An XML Framework for Agent-based Ecommerce, Communications of the ACM 42(3), 106-114 (1999).

3. Workflow Management Coalition, Workflow Standard - Interoperability: Wf-XML Binding (May, 2000), http://www.wfmc.org/standards/docs.

4. W.M.P. Vander Aalst, Process-Oriented Architectures for Electronic Commerce and Interorganizational Workflow, Information Systems 24(8), 639-671 (1999).

5. M. Girardot and N. Sundaresan, Millau: An Encoding Format for Efficient Representation and Exchange of XML over the Web, Computer Networks 33(6), 747-765 (2000).

6. J. Korhonen, L. Pajunen, and J. Puustjrvi, Automatic Composition of Web Service Workflows Using a Semantic Agent, Proceedings of the IEEE/WIC International Conference on Web Intelligence, 2003, p.566.

7. G. Piccinelli, W. Emmerich, C. Zirpins, K. Schütt, Web Service Interfaces for InterOrganisational Business Processes: An Infrastructure for Automated Reconciliation, Proceedings of the 6th International Enterprise Distributed Object Computing Conference, 2002, pp. 285-292.

8. Workflow Management Coalition, WfMC-TC-1016-P, Process Definition Interchange Process Model, 1999.

9. Workflow Management Coalition, WFMC-TC-1009 - Specification, Workflow Management Application Programming Interface Specification, 1998.

10. M. B. Blake, Object-Oriented Modeling Approaches to Agent-Based Workflow Services, 2004.

11. R. Lara, L. Lausen, S. Arroyo, J. Bruijn, and D. Fensel, Semantic Web Services: Descriptions, Requirements and Current Technologies, International Workshop on Agents and Semantic Web Services (ICEC2003), Pittsburgh, PA, 2003.

12. M.P. Singh, B. Yu, and M. Venkatraman, Community-Based Service Location, $C A C M$ 44(4), 49-54, (2001). 
13. UDDI Specification 2.04 (2002), http://uddi.org/pubs/ProgrammersAPI-V2.04-Published20020719.pdf.

14. W.M.P. Van der Aalst, Don't Go with the Flow: Web Services Composition Standards Exposed, IEEE Intelligent, 2003.

15. Web Services (2002), http://www.w3.org/2002/ws/desc/.

16. A.B. Williams, A. Padmanabhan, and M.B. Blake, Local Consensus Ontologies for B2Boriented Service Composition, Proceedings of the AAMAS2003, 2003.

17. L. Zeng, A. Ngu, B. Benatallah, and M. O'Dell, An Agent-based Approach for Supporting Inter-enterprise Workflows. In the Proceedings of the 12th Australasian Database Conference, IEEE Society, Gold Coast, Australia, 2001. 